\title{
eJRIEPS
}

Ejournal de la recherche sur l'intervention en éducation physique et sport

$26 \mid 2012$

Varia

\section{Une histoire des savoirs en natation acquis chez une population d'enseignant-e-s d'EPS entre 1945 et 1995}

\section{Emmanuel Auvray}

\section{(2) OpenEdition}

1 Journals

Édition électronique

URL : http://journals.openedition.org/ejrieps/3323

DOI : $10.4000 /$ ejrieps.3323

ISSN : 2105-0821

Éditeur

ELLIADD

Référence électronique

Emmanuel Auvray, «Une histoire des savoirs en natation acquis chez une population d'enseignant-e-s d'EPS entre 1945 et 1995 », eJRIEPS [En ligne], 26 | 2012, mis en ligne le 01 avril 2012, consulté le 10 décembre 2020. URL : http://journals.openedition.org/ejrieps/3323 ; DOI : https://doi.org/10.4000/ ejrieps.3323

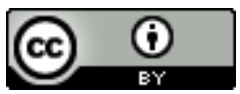

La revue eJRIEPS est mise à disposition selon les termes de la Creative Commons Attribution 4.0 International License. 
eJRIEPS 26 avril 2012

Une histoire des savoirs en natation acquis chez une population d'enseignante-s d'EPS entre 1945 et 1995

\section{Emmanuel Auvray}

Centre de Recherche et d'Innovation sur le Sport, EA 647, Université Lyon 1

\section{Résumé}

Cette étude vise à rendre compte chez une population d'enseignant-e-s d'EPS de l'évolution (1945-1995) des savoirs natatoires acquis durant leur socialisation primaire et secondaire. À partir d'une enquête par questionnaire, complétée par des témoignages écrits, et des sources privées ou semi-officielles, il en ressort en fonction des entités épistémiques fréquentées des permanences et des disparités curriculaires en natation. Ces écarts semblent s'être atténués au fil du temps du fait de l'amélioration des conditions locales de formation initiale. Les savoirs appris par le bais de la formation professionnelle continue, des formations fédérales et de l'autoformation ont globalement gravité autour des aspects historiques, réglementaires et technologiques liés aux nages sportives et sur l'hégémonique conception didactique de $R$. Catteau axée sur l'apprentissage du triptyque équilibre-propulsion-respiration. Nos résultats nous questionnent sur l'égalité des chances de réussite aux concours de recrutement d'enseignant-e-s d'EPS eu égard aux savoirs dispensés dans les structures de formation et sur l'impact des savoirs natatoires acquis et leurs rapports sur les pratiques pédagogiques de terrain développées au cours de leur carrière.

\section{Introduction}

En France, qu'il s'agisse du recrutement par concours du personnel enseignant d'éducation physique et sportive (EPS) ou de l'enseignement de l'EPS, le législateur a toujours défini au cours du XXe siècle ses exigences curriculaires à l'échelon national. S'agissant de l'exercice de cette profession elle repose, comme chez les autres enseignants (Clermont Gauthier et al., 1997), sur un ensemble de savoirs et d'éléments culturels acquis en fonction des parcours biographiques personnels et professionnels façonnant alors les identités professionnelles (Dubar, 2000 ; RouxPerez, 2011). Or, entre 1945 et 1995, l'histoire des formations initiales menant au 


\section{eJRIEPS 26 avril 2012}

métier d'enseignant d'EPS a été marquée par un processus d'universitarisation et d'homogénéisation (Michon, 1983 ; Mierzejewski, 2005) des études sous-tendu par des enjeux politiques, syndicaux et idéologiques. Ainsi, en l'espace d'un demi-siècle, nous sommes passés de la pluralité à la singularité des formations initiales en EPS (Néaumet, 1992) avec la mise en place en 1982 de la $74^{\mathrm{e}}$ section universitaire en Sciences et Techniques des Activités Physiques et Sportives (STAPS) et la suppression en 1984 du recrutement par concours des professeur-e-s adjoint-e-s d'EPS formé-e-s, comme les maîtres d'EPS auparavant, dans les Centres Régionaux d'Education Physique et Sportive (CREPS). De nos jours, seul un parcours d'études en STAPS donne le droit de pouvoir se présenter aux concours externes ou internes du Certificat d'Aptitude au Professorat d'EPS (CAPEPS) ou de l'agréation d'EPS pour accéder au statut d'enseignant titulaire. Ces lignes d'évolution ont manifestement pu faire évoluer la nature des savoirs transmis aux étudiant-e-s en EPS ainsi que leurs conditions d'acquisition. C'est pourquoi, entre 1945 et 1995, la nature des savoirs réellement transmis dans les structures qui ont préparé des étudiant-e-s au CAPEPS, à la maîtrise d'EPS, puis au Professorat Adjoint (PA) d'EPS, a pu varier en raison d'une part, d'un ensemble de facteurs liés au recrutement et à l'origine sociale et culturelle des étudiant-e-s (Defrance, 1978 ; Michon, 1982), à la durée des études, aux enjeux de formation et aux contenus exigés et, d'autre part, à la disparité à l'échelon local des moyens humains et matériels propres aux différents centres de formation. En outre, les enseignant-e-s d'EPS ont généralement appris des savoirs utiles à l'exercice de leur métier en amont de leurs études dans le champ notamment des activités physiques sportives et artistiques (APSA) soit en tant que pratiquant ou/et encadrant, ainsi que durant leur carrière par le biais de l'auto-formation et de la formation professionnelle continue (FPC) (Attali, 2006). Dès lors, en fonction de la nature des cheminements biographiques, les enseignant-e-s d'EPS ont pu se doter d'un ensemble de savoirs et entretenir avec ceux liés aux APSA différents rapports allant par exemple de l'appétence au déplaisir de la pratique. Cette étude porte donc sur la formalisation des savoirs acquis sur l'activité natation chez une population relativement hétérogène d'enseignant-e-s d'EPS $(n=143)$ au cours de leur socialisation primaire et secondaire. II s'agira de voir comment les parcours biographiques personnels et professionnels, du point de vue des entités épistémiques fréquentées en tant que 
eJRIEPS 26 avril 2012

support de diffusion de savoirs (Lahire, 1998), ont pu impacter la nature des savoirs natatoires acquis avant, pendant et après les études en EPS.

\section{Les savoirs pratiques, théoriques, d'action et d'expérience en EPS, objets d'histoire}

D’une manière générale, la formation initiale et continue des enseignant-e-s repose sur l'acquisition d'un ensemble de savoirs dans l'optique de former un professionnel maîtrisant des savoirs disciplinaires et des savoirs utiles à leur transmission au moment d'appliquer le curriculum formel ou prescrit. La formation initiale des enseignant-e-s s'effectue, de manière implicite ou explicite, selon un modèle type daté et situé (Postic, 1977) en référence aux exigences fixées par État recruteur. Plus vaste que la connaissance, le savoir renvoie à un ensemble d'informations, d'énoncés, d'actions, disponibles dans un domaine donné. Chez les enseignant-e-s, les savoirs s'expriment par la capacité à conduire des actions finalisées dans des situations d'apprentissage complexes en raison d'un ensemble de variables médiatrices dont dépendent alors les actions professionnelles liées à l'acte d'enseigner des savoirs à des élèves. Dans le domaine de l'enseignement scolaire, Clermont Gauthier et al. ont formalisé le réservoir des savoirs que les enseignants «mettent en action pour répondre à certaines demandes précises liées à des situations concrètes d'enseignement » (1997, p. 20).

Tableau I. Le réservoir des savoirs selon Clermont Gauthier et al. (1997, p. 20).

\begin{tabular}{|l|l|l|l|l|l|}
\hline Savoirs & Savoirs & Savoirs & Savoirs & Savoirs & Savoirs \\
\hline Lisciplinaires & Curriculaires & Des sciences & De la tradition & D'expérience & D'action pédagogique \\
& Le programme & de l'éducation & pédagogique & La référence privée & base des connaissances \\
& & La coutume & & La en enseignement ou la \\
& & & & \\
\hline
\end{tabular}

S'agissant plus spécifiquement des enseignant-e-s d'EPS et de la natation, nous avons cherché à identifier, à côté des autres savoirs, ceux qui avaient été acquis sur cette activité physique et sportive (APS) durant les phases de socialisation primaire et secondaire en fonction des choix de trajectoires biographiques personnelles et 


\section{eJRIEPS 26 avril 2012}

professionnelles dans et hors du champ de l'EPS. Pour organiser cette formalisation, nous avons au préalable énoncé quatre types de savoirs en natation.

Les premiers, les savoirs pratiques renvoient aux expériences motrices vécues en tant que nageur, plus ou moins expert dans cette activité. Ils correspondent aux savoirs qui ont été incorporés au cours de séances pratiques durant la socialisation primaire et/ou secondaire. Ces savoirs s'expriment par la maîtrise de compétences et de techniques corporelles hautement spécialisées et finalisées par l'atteinte d'un but tel que la recherche de performance chronométrique. Ces savoirs pratiques incorporés et hérités (Dubar, 2000) durant notamment les études en EPS posent, chez les enseignant-e-s d'EPS, en filigrane la question de leurs rapports aux savoirs à enseigner aux élèves entre appétence et refus, entre compétence et ignorance, entre accord et désaccord en fonction de leur vécu, de leur degré d'expertise et de leur ressenti personnel. Les seconds, les savoirs théoriques, portent sur l'analyse de l'activité natation course, en tant que pratique sociale, au travers de ces aspects historiques, réglementaires, bio-mécaniques, technologiques et anthropologiques. Quant aux savoirs d'action professionnelle, ils sont liés à la pédagogie de la natation, c'est-à-dire à la manière d'organiser et de conduire en vis-à-vis réel avec des élèves un enseignement ou un entraînement en natation. Nous incluons dans ces savoirs d'action ceux qui relèvent de la didactique de la natation autour de la détermination, de la différenciation, de la hiérarchisation et de l'évaluation des contenus à transmettre aux élèves avant et après l'acte d'enseigner. Enfin, les savoirs d'expérience reposent sur le fait qu'ils «introduisent un rapport particulier à l'habitude » (Clermont Gauthier et al., 1997, p. 23) dans la mesure où l'on apprend de ses expériences. Ces derniers permettent une jurisprudence, c'est-à-dire une référence privée qui s'établit dans le répertoire des savoirs d'expérience de chacun utiles à l'enseignement des APSA en EPS.

Les enseignant-e-s d'EPS de la population ici étudiée ont vraisemblablement acquis, sur la natation et son enseignement, un ensemble de savoirs pratiques, théoriques, d'action et d'expérience dont la nature a pu varier en fonction des époques et des entités épistémiques fréquentées dans et hors de leur univers professionnel. Ce faisant, nous avons différencié trois profils d'expertise $\left(1^{\mathrm{er}}, 2^{\mathrm{ème}}, 3^{\mathrm{ème}}\right)$ et d'expérience en natation eu égard à leurs choix de trajectoires biographiques. Nous avons pris en compte, pour l'activité natation, leurs profils de pratique, la nature de leurs formations 
eJRIEPS 26 avril 2012

et de leurs qualifications, leurs expériences d'encadrement. Au final, nous avons retenu les trois profils d'expérience et d'expertise suivants : $1^{\text {er }}$ profil : simple nageur et parfois titulaire du diplôme de maître nageur sauveteur (MNS) ayant été obtenu par équivalence avant $1965 ; 2^{\text {ème }}$ profil : nageur de compétition, optionnaire natation durant les études en EPS et titulaire du diplôme de MNS obtenu entre 1965 et 1983 puis du Brevet d'État d'Éducateur des Activités de la Natation (BEESAN, option natation, $1^{\text {er }}$ degré) à compter de 1986 ; $3^{\text {ème }}$ profil : nageur de compétition, optionnaire durant les études en EPS, titulaire du diplôme de MNS ou du Brevet d'état d'entraîneur de natation $1^{\mathrm{er}}, 2^{\text {ème }}$ ou $3^{\text {ème }}$ degré ou du BEESAN, entraîneur de clubs civils de natation et/ou au sein du sport scolaire, formateur pour l'activité natation soit au sein de l'univers professionnel de l'EPS ou/et dans le champ sportif pour les diplômes suivants : MNS, Brevet d'état d'entraîneur, BEESAN.

\section{Le témoignage d'acteurs ordinaires de l'EPS, une plus value pour l'histoire}

Si l'historiographie de l'EPS est manifestement riche de travaux sur la parole d'autorité que sont les textes officiels et sur la parole «sacrée » au travers des conceptions de l'EPS, en revanche, il existe moins de recherches historiques sur le contenu concret des formations initiales et continues du personnel enseignant d'EPS (Terret, 1998 ; Collinet \& Taled, 2003). Selon nous, ce manque de données historiques sur la nature des savoirs qui ont été réellement transmis et acquis chez les enseignant-e-s d'EPS tient d'une part, à l'opacité et au caractère privé de l'acte formatif révolu et, d'autre part, à la difficulté de trouver des sources et des témoins volontaires et relativement âgés. C'est pourquoi, pour recueillir des données sur des savoirs natatoires acquis avant, pendant, et après des études en EPS, nous avons eu recours à la méthodologie du questionnaire et du témoignage écrit complétée par des sources indiciaires privées ou semi-officielles auprès d'enseignant-e-s âgé-e-s de 43 à 87 ans. Cette démarche donne la possibilité de pouvoir contrôler les biais mémoriels inhérents à ce genre d'étude et de limiter la subjectivité des acteurs et les oublis. Car, outre que les sujets ont une certaine propension à produire selon Franco Ferrarotti (1990, p. 30) « une historicité non historique », la mémoire des acteurs peut être affectée par des oublis volontaires ou involontaires (Ricœur, 2000) et soumise à des influences collectives institutionnelles, pédagogiques et corporatives. En effet, selon Maurice Halbwachs (1994), la mémoire des groupes professionnels est en partie modelée par des normes et des repères implicites et/ou explicites qui 


\section{eJRIEPS 26 avril 2012}

infléchissent la mémoire des sujets en leur donnant un certain sens en fonction d'un système de valeurs, de croyances et d'idées propres au groupe professionnel d'appartenance. Ainsi, chaque groupe professionnel se définit sur un plan identitaire à partir d'un idéal type, daté et situé, qui sous-tend un noyau dur de finalités, de valeurs, d'objectifs et d'actions professionnelles (Legras, 1999) pour l'essentiel partagé par l'ensemble des acteurs d'une même corporation (Roux-Perez, 2011). Ce faisant, nous avons cherché à établir, selon le principe de la mise en récurrence d'items (Bertaux, 1997, Robert, \& Bouillaguet, 1997), une périodisation à l'endroit de l'évolution (1945-1995) des savoirs natatoires acquis chez une population hétérogène d'enseignant-e-s d'EPS ayant vécu différentes trajectoires biographiques personnelles et professionnelles. Si la période d'étude a été volontairement bornée de 1945 à 1995, c'est parce que premièrement les acteurs les plus âgés que nous avons pu questionner ont débuté leurs études en EPS en 1945 ; et que concernant la borne supérieure de 1995, nous n'avons pas souhaité pour des raisons d'objectivité historique analyser - dans une autre partie de cette étude (Auvray, 2011) - des pratiques pédagogiques encore encadrées, au moment de démarrer ce travail de recherche, par les textes officiels parus à compter de 1996 (Collège). En outre, l'historicité des acteurs étant par nature non historique (Ferrarotti, 1990), si nous avions porté notre attention sur une histoire trop au présent, cela aurait sans doute nuit à l'objectivité des propos déclarés dans les questionnaires et leurs relances.

\section{Des maîtres, des professeurs-adjoints et des certifiés d'EPS}

La population ici étudiée est volontairement composée d'enseignant-e-s d'EPS $(n=143)$ ayant eu des trajectoires biographiques à la fois communes et différentes tant au niveau des entités épistémiques fréquentées que de la contextualisation de leur agir professionnel au collège et/au lycée. Si nous avons délibérément choisi de sonder des enseignant-e-s d'EPS rattaché-e-s à trois statuts administratifs différents, c'est dans l'optique de pouvoir valider ou non l'hypothèse de travail suivante : de manière synchronique et diachronique, on suppose que lorsque les trajectoires biographiques d'enseignant-e-s d'EPS seraient relativement semblables du point de vue de la formation initiale, des concours de recrutement passés, de l'expérience et de l'expertise dans l'activité natation, elles auraient influencé de manière identique la nature de leurs savoirs en natation, malgré la variabilité et la contextualisation des structures et des formes de formation vécues en tant que succession d'actions 


\section{eJRIEPS 26 avril 2012}

formatives datées et situées. Indiquons les principales caractéristiques de la population étudiée.

Tableau II. Caractéristiques de la population étudiée.

\begin{tabular}{|c|c|c|c|c|c|c|c|}
\hline $\begin{array}{l}\text { Structures de } \\
\text { formation initiale }\end{array}$ & $\mathrm{H}$ & $F$ & $\begin{array}{l}\text { Le ou la plus } \\
\text { âgé(e) né(e) en }\end{array}$ & $\begin{array}{l}\text { Le ou la plus } \\
\text { jeune né(e) en }\end{array}$ & $\begin{array}{c}\text { Certifié(e) } \\
\text { d'EPS }\end{array}$ & \begin{tabular}{|c|} 
Maître(sse) \\
d'EPS
\end{tabular} & $\begin{array}{c}\text { Professeur(e) } \\
\text { adjoint(e) }\end{array}$ \\
\hline ENSEP & 25 & 3 & né en 1923 & née en 1949 & 28 & & \\
\hline IREPS & 16 & 3 & né en 1933 & né en 1959 & 19 & & \\
\hline CREPS & 31 & 19 & né en 1932 & né en 1960 & 24 & 12 & 14 \\
\hline $\begin{array}{l}\text { UER EPS et } \\
\text { UFR STAPS }\end{array}$ & 30 & 16 & né en 1952 & né en 1967 & 46 & & \\
\hline Totaux & 102 & 41 & & & 117 & 12 & 14 \\
\hline
\end{tabular}

(Pour $\mathrm{H}$, lire « hommes », pour $\mathrm{F}$ lire « femmes »)

En ce qui concerne la représentativité de notre échantillon, il apparaît clairement que les professeurs certifiés de sexe masculin $(n=115)$, formés au sein des CREPS, des Instituts Régionaux d'EPS (IREPS), des Écoles Normales Supérieures d'EPS (ENSEPS), des Unités d'Enseignement et de Recherche en EPS (UER EPS) ou des Unités de Formation et de Recherche en Sciences et Techniques des APS (UFR STAPS), sont nettement plus représentés que les maîtres $(n=12)$ et les professeurs adjoints (PA) d'EPS ( $n=12$ ) exclusivement issus, eux, des CREPS. S'agissant de la répartition entre les hommes et les femmes notre échantillon indique que, par rapport à l'échelon national, les enseignants (70\%) sont nettement plus représentés que les enseignantes (30\%). Effectivement, la note d'information du Ministère de Éducation Nationale $n^{\circ}$ 97-11 (1997) indique au niveau nationa I une répartition entre le nombre d'enseignants d'EPS hommes $(H)$ et femmes $(F)$ de l'ordre de $53 \%(H)$ pour $47 \%$ $(F)$, pour un total d'environ 33500 enseignant-e-s toutes catégories statutaires confondues. Par ailleurs, comme l'ont souligné certains travaux universitaires (Defrance, 1978 ; Michon, 1982), l'origine sociale et culturelle des enseignant-e-s d'EPS de la population étudiée a pu peser sur la construction de leurs savoirs en natation entre les certifiés, les maîtres et les PA d'EPS en raison de leur capital corpo-culturel initial. Au final, la population étudiée n'est que partiellement représentative de l'ensemble de la population des enseignant-e-s d'EPS qui ont exercé ce métier entre 1945 et 1995 . Que pouvons-nous dire de la ventilation des 


\section{eJRIEPS 26 avril 2012}

trois profils d'expertise et d'expérience acquis en natation en fonction de leur identité sexuée et de leur origine statutaire ?

Tableau III. Ventilation des enseignant-e-s d'EPS et degré d'expertise et d'expérience en natation (1945-1995).

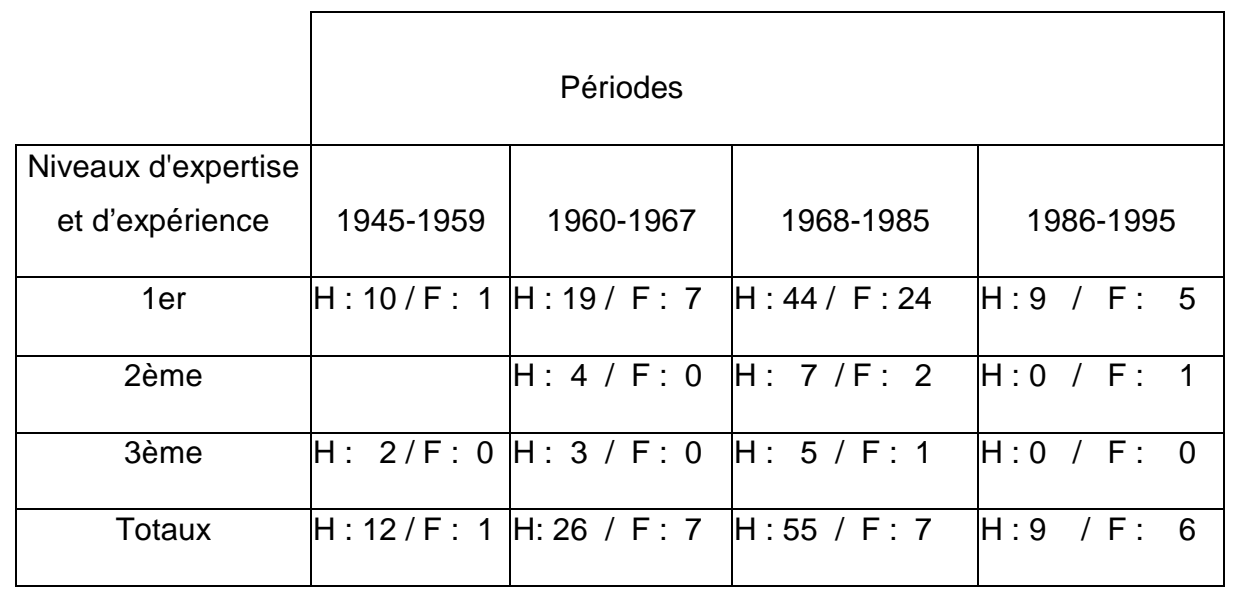

Pour ce qui relève de la répartition de l'identité sexuée, le tableau III montre qu'environ $58 \%$ des hommes et $23 \%$ des femmes ont le $1^{\text {er }}$ profil d'expertise et d'expérience en natation, environ $7,6 \%$ des hommes et $2 \%$ des femmes ont le $2^{\text {ème }}$ profil, et enfin $6,3 \%$ des hommes et des $1,4 \%$ des femmes ont le $3^{\text {ème }}$ profil. Autrement dit, entre 1945 et 1995, les écarts de répartition des différents profils d'expertise et d'expérience dans l'activité natation, selon les périodes ici considérées, sont à peu près identiques entre les hommes et les femmes sachant toutefois que les hommes sont au départ nettement plus représentés (71\%) que les femmes dans notre échantillon.

Du côté de la répartition des trois profils d'expertise et d'expérience en fonction de l'origine statutaire et des études en EPS (1945-1995), nous pouvons indiquer que, sauf chez les maîtres d'EPS, nous trouvons des enseignant-e-s d'EPS certifié-e-s ou des PA d'EPS qui ont soit le $1^{\mathrm{er}}$ profil, le $2^{\text {ème }}$ ou le $3^{\text {ème }}$. Parmi les trois catégories statutaires analysées, nous avons continuellement, entre 1945 et 1995, des enseignant-e-s certifié-e-s, des maîtres-ses et des PA d'EPS qui possèdent logiquement le $1^{\mathrm{er}}$ profil d'expertise et d'expertise en natation puisque cette APS a toujours fait partie des épreuves physiques obligatoires pour entrer en formation initiale et des programmes des épreuves physiques des concours de recrutement: CAPEPS, maîtrise et PA d'EPS. Toutefois, de manière plus ciblée, on note que chez 


\section{eJRIEPS 26 avril 2012}

les maîtres d'EPS aucun ne dispose du $2^{\text {ème }}$ profil mais seulement un homme qui, pour la période $1959-1967$, a le $3^{\text {ème }}$ profil et 11 le $1^{\text {er }}$ profil, soit $91,5 \%$. Chez les PA d'EPS, nous avons 11 enseignants, 5 hommes et 6 femmes, soit $78,5 \%$ qui ont le $1^{\text {er }}$ profil ; 1 homme a le $2^{\text {ème }}$ profil et un homme et une femme ont le $3^{\text {ème }}$ profil, soit 14\%. Proportionnellement, il y a donc plus de spécialistes de natation chez les PA d'EPS que chez les maîtres d'EPS. Ce constat renvoie au fait que les étudiant-e-s sélectionné-e-s pour devenir PA d'EPS étaient davantage sélectionnés et formés dans les CREPS en fonction de leur spécialité sportive (Hurtebize, 1981) pratiquée en amont de leurs études en EPS que les élèves-professeur-e-s, voire les maîtres d'EPS. Ainsi, on peut supposer que les maîtres-ses d'EPS et les PA d'EPS étaient plus spécialisés dans leur option sportive que les futurs professeur-e-s certifié-e-s eu égard aux modalités de recrutement initial, aux contenus des études et aux épreuves des concours (Néaumet, 1992). Si l'on compare la répartition des différents profils d'expertise et d'expérience dans l'activité natation entre les certifié-es, les maîtresses, et les PA d'EPS, on remarque qu'ils sont toujours logiquement plus nombreux à posséder le $1^{\text {er }}$ profil que le $2^{\text {ème }}$ ou le $3^{\text {ème, }}$ quelle que soit leur catégorie administrative initiale. Nonobstant, on note qu'il y a autant voire davantage d'enseignants hommes que femmes qui sont titulaires du $2^{\text {ème }}$ ou du $3^{\text {ème }}$ profil sans doute pour des raisons socio-culturelles liées aux mentalités et au métier d'entraîneur ainsi qu'à la disponibilité temporelle entre les hommes et les femmes.

\section{La construction sociale des savoirs et des logiques d'action : entre formations initiales, univers professionnel et champ sportif (1945-1995)}

Les enseignant-e-s d'EPS de la population ici analysée ont tous acquis, sur la natation et son enseignement, un ensemble de savoirs pratiques, théoriques, d'action et d'expérience au cours de leur socialisation primaire et secondaire en fonction des entités épistémiques fréquentées en tant que support de diffusion de savoirs. Voyons maintenant, de manière plus précise, quels ont pu être ces savoirs eu égard à leurs parcours biographiques dans et hors du champ de l'EPS ?

5. 1. Les formations initiales en EPS: quels savoirs aquatiques exigés et réellement appris?

Entre 1945 et 1995, le législateur a toujours imposé dans les épreuves du CAPEPS, de la maîtrise d'EPS, puis du PA d'EPS, que les candidat-e-s fassent montre entre 


\section{eJRIEPS 26 avril 2012}

autres de leurs savoirs pratiques, théoriques, d'action et d'expérience à l'endroit de l'activité natation tant au niveau des épreuves écrites, orales que pratiques et pédagogiques. Notons que l'activité natation, à côté de l'athlétisme, de la gymnastique sportive et des sports collectifs, a occupé au moins durant la seconde moitié du XXe siècle une place cardinale dans tous les programmes des concours de recrutement d'enseignant-e-s d'EPS et dans les textes officiels qui ont encadré l'enseignement de l'EPS en raison de ses vertus prophylactiques et de la nécessité d'apprendre aux élèves au minimum à nager pour se sauver (Pelayo \& Terret, 1994). Par ailleurs, l'évolution des savoirs natatoires exigés en natation au niveau du CAPEPS, de la maîtrise d'EPS puis du PA d'EPS a plutôt suivi ceux liés à la natation sportive extrascolaire, en atteste la présence d'épreuves chronométrées et des démonstrations techniques en quatre nages dès les années cinquante. En outre, en liaison avec les pratiques physiques des français en matière de natation (Dourdin, 1942 ; INSEP, 1987), on observe que le législateur a continuellement visé à ce que les enseignant-e-s d'EPS soient formé-e-s (1945-1995), durant leurs études, pour donner à leurs élèves les moyens techniques pour qu'ils puissent a minima nager pour ne pas se noyer voire pour s'entretenir physiquement en pratiquant la brasse et/ou le crawl. Pour formaliser l'évolution des savoirs natatoires qui ont été exigés, entre 1945 et 1995, par le législateur, nous avons sondé le Bulletin Officiel de l'Éducation nationale ; et pour identifier ceux concrètement enseignés (1945-1995) à des étudiant-e-s en EPS et acquis durant leur carrière, nous avons utilisé un questionnaire complété par des témoignages écrits et des sources privées comme des notes de cours, des exposés etc. De cette investigation diachronique et descriptive, nous montrerons qu'il en ressort des permanences et quelques changements quant à l'évolution de la nature des savoirs natatoires exigés par l'État recruteur et réellement dispensés dans les centres de formation et appris par le biais de la formation professionnelle continue et l'auto-formation. Donnons tout d'abord des repères sur l'évolution des savoirs natatoires exigés par les concours de recrutement d'enseignant-e-s d'EPS (1945-1995) : CAPEPS, maîtrise d'EPS, PA d'EPS. 
Tableau IV. Évolution des savoirs natatoires exigés au niveau du CAPEPS (19451995).

\begin{tabular}{|c|c|c|c|}
\hline & Savoirs pratiques & Savoirs théoriques & Savoir d'action \\
\hline $\begin{array}{l}\text { CAPEPS } \\
1945-1965\end{array}$ & $\begin{array}{c}\text { 50m chronométré au choix parmi } \\
\text { brasse, } \\
\text { crawl, dos et démonstrations } \\
\text { techniques } \\
\left(1^{\text {er }} \text { et } 2^{\text {ème }} \text { partie }\right)\end{array}$ & $\begin{array}{l}\text { Règlements et techniques } \\
\qquad\left(1^{\mathrm{er}} \text { et } 2^{\text {ème }} \text { partie }\right)\end{array}$ & $\begin{array}{l}\text { Apprentissage d'un geste sportif, leçon } \\
\text { de natation élémentaire ou sportive et } \\
\text { démonstrations (épreuve de classement) }\end{array}$ \\
\hline $\begin{array}{r}\text { CAP } \\
1966 \\
\end{array}$ & $\begin{array}{c}100 \text { chronométré au choix parmi } \\
\text { brasse, crawl, } \\
100 \text { chronométré au choix NL, dos, } \\
\text { papillon, } \\
400 \mathrm{NL}, 200 \text { brasse, démonstrations } \\
\text { plongeons, water-polo (JG), natation } \\
\text { synchronisée (JF) démonstrations/ } \\
\text { corrections }\end{array}$ & $\begin{array}{c}\text { Règlements et techniques des } \\
\text { exercices physiques et sportifs à } \\
\text { l'écrit et à l'oral } \\
\left(2^{\mathrm{ème}} \text { partie, probatoire }\right)\end{array}$ & $\begin{array}{l}\text { Leçon de natation élémentaire } \\
\text { ou sportive avec démonstrations } \\
\text { et interrogation sur les techniques } \\
\left.\text { ( } 2^{\text {eme }} \text { partie, classement }\right)\end{array}$ \\
\hline $\begin{array}{l}\text { CAPEPS } \\
1971-1980\end{array}$ & \begin{tabular}{|} 
1 épreuve chronométrée au choix \\
parmi \\
$100 \mathrm{~m} \mathrm{NL}, 400 \mathrm{NL}, 1500 \mathrm{NL}, 100 \mathrm{~m}$, \\
$200 \mathrm{~m}$ \\
dos, brasse ou papillon, $200 \mathrm{~m} 4$ nages \\
(épreuves de classement)
\end{tabular} & $\begin{array}{l}\text { Aspects techniques } \\
\text { et réglementaires } \\
\text { (épreuves de classement) }\end{array}$ & $\begin{array}{c}\text { Polyvalence APS : programme } \\
\text { d'enseignement, progression et } \\
\text { démonstrations } \\
\text { Séance de natation en fonction de : âge, } \\
\text { niveau, difficultés rencontrées. } \\
\text { Option APS : séance de natation : } \\
\text { enseignement, initiation, entraînement. } \\
\text { Aspects techniques didactiques et } \\
\text { pédagogiques } \\
\end{array}$ \\
\hline $\begin{array}{c}\text { CAPEPS } \\
1981-1989\end{array}$ & \begin{tabular}{|c|} 
Epreuve d'option : 1 épreuve \\
chronométrée au choix parmi en NL \\
$100,400,800$ (JF), ou $200 \mathrm{~m}, 4$ nages, \\
200 ou 400 (admissibilité) \\
Epreuve de polyvalence : 1 épreuve \\
chronométrée au choix parmi: 100 \\
brasse, 100 NL, 100 papillon, 2004 \\
nages (admission)
\end{tabular} & $\begin{array}{l}\text { Aspects techniques, } \\
\text { réglementaires et historiques } \\
\text { (admission) }\end{array}$ & \begin{tabular}{|c} 
Option APS : composition d'une séance \\
Polyvalence APS : programme, \\
programmation, progression maîtrise du \\
milieu aquatique, enseignement des \\
4 nages, séance natation, initiation. \\
Sauvetage (admission)
\end{tabular} \\
\hline $\begin{array}{l}\text { CAPEPS } \\
1990-1995\end{array}$ & $\begin{array}{l}\text { 100m chronométré au choix, parmi } \\
\text { brasse, } \\
\text { crawl, dos, papillon, 200m } 4 \text { nages } \\
\text { (admission) }\end{array}$ & $\begin{array}{l}\text { Aspects techniques, } \\
\text { réglementaires et historiques } \\
\text { (admissibilité et admission) }\end{array}$ & $\begin{array}{c}\text { Oral } 1 \text { : concevoir, construire et conduire } \\
\text { une séance de natation (cycle) suite à un } \\
\text { stage en situation. } \\
\text { Oral } 2 \text { : aspects techniques, didactiques } \\
\text { pédagogiques du débutant au nageur } \\
\text { confirmé (admission) }\end{array}$ \\
\hline
\end{tabular}


Tableau V. Évolution des savoirs natatoires exigés au niveau de la maîtrise d'EPS et du PA d'EPS (1945-1984).

\begin{tabular}{|c|c|c|c|}
\hline & Savoirs pratiques & Savoirs théoriques & Savoirs d'action \\
\hline Maîtrise d'EPS & $\begin{array}{c}\text { 50m chronométré au choix parmi } \\
\text { brasse, } \\
\text { crawl, dos et démonstrations } \\
\text { techniques } \\
\text { (M1 et M2) }\end{array}$ & $\begin{array}{l}\text { Règlements et } \\
\text { techniques(M2) }\end{array}$ & $\begin{array}{l}\text { Apprentissage d'un geste sportif, leçon } \\
\text { d'initiation sportive natation élémentaire } \\
\text { ou sportive avec démonstrations (M2) }\end{array}$ \\
\hline $\begin{array}{c}\text { Maîtrise d'EPS } \\
\text { 1960-1969 }\end{array}$ & \begin{tabular}{|c|}
$50 m$ chronométré au choix nage \\
libre ou \\
brasse (M1) 50m chronométré au \\
choix \\
nage libre ou brasse ou dos crawlé \\
(M2)
\end{tabular} & $\begin{array}{l}\text { Règlements et techniques } \\
\text { (M2) } \\
\text { (brasse ou crawl ou dos } \\
\text { crawlé) }\end{array}$ & $\begin{array}{l}\text { Apprentissage d'un geste sportif, } \\
\text { leçon de natation élémentaire } \\
\text { ou sportive avec démonstrations (M2) }\end{array}$ \\
\hline $\begin{array}{c}\text { Maîtrise d'EPS } \\
1970-1972 \\
\end{array}$ & $\begin{array}{c}\text { 50m chronométré nage libre (M1) } \\
\text { Démonstrations techniques : } \\
\text { brasse, } \\
\text { crawl, dos crawlé, plongeon de } \\
\text { départ et virage } \\
\end{array}$ & $\begin{array}{c}\text { Règlements et } \\
\text { techniques (M2) } \\
\text { (brasse ou crawl ou dos } \\
\text { crawlé) }\end{array}$ & $\begin{array}{c}\text { Apprentissage d'un geste sportif, } \\
\text { leçon de natation élémentaire } \\
\text { ou sportive avec démonstrations (M2) }\end{array}$ \\
\hline $\begin{array}{c}\text { Maîtrise d'EPS } \\
\begin{array}{c}\text { 1973-1975 } \\
\text { et } \\
\text { PA d'EPS } \\
1975-1984\end{array}\end{array}$ & $\begin{array}{c}\text { 50m chronométré au choix parmi } \\
\text { brasse, } \\
\text { crawl, dos ou papillon (M1, } \\
\text { polyvalence } \\
\text { choix } \\
\text { APS). Epreuve chronométrée au } \\
\text { parmi en NL 100m, 200m, } 800 \mathrm{~m} \\
\text { dos, } \\
\text { brasse et papillon : } 100 \mathrm{~m}, 200 \mathrm{~m} \\
200 \mathrm{~m} 4 \text { nages et des } \\
\text { démonstrations } \\
\text { commentées (option APS, M2) }\end{array}$ & $\begin{array}{l}\text { Techniques et règlements } \\
\text { Fonction d'officiel et } \\
\text { organiser } \\
\text { et gérer une compétition (M2) }\end{array}$ & $\begin{array}{l}\text { Etablir un programme, } \\
\text { une progression, une séance } \\
\text { Etablir un entraînement (M2) }\end{array}$ \\
\hline
\end{tabular}

De l'analyse des tableaux IV et $\mathrm{V}$, il en ressort, concernant la nature des savoirs pratiques, théoriques et d'action en natation exigés par l'État recruteur (1945-1995) les lignes d'évolution suivantes. Premièrement, on peut écrire que s'agissant des savoirs pratiques les candidat-e-s au CAPEPS, à la maîtrise d'EPS et au PA d'EPS ont dû maîtriser (1945-1995) au minimum, à côté de l'épreuve obligatoire et éliminatoire de sauvetage aquatique, une technique de nage sportive pour nager le plus vite possible et réglementairement lors des épreuves de parcours chronométré en natation; et, au moins, deux autres nages sportives entre 1945 et 1959, puis trois, pour les démonstrations techniques demandées entre 1960 et 1970 . Nous pouvons ajouter qu'avec la mise en place des options et des polyvalences d'APS à compter des sessions de 1971, on a vu apparaître dans les programmes des épreuves physiques de natation la quasi-totalité des courses habituellement programmées par la Fédération Internationale de Natation Amateur (FINA). Ainsi, au début des années 70 , les distances exigées et le choix des épreuves de spécialité proposées aux élèves professeurs et aux élèves-maitres ont correspondu quasiment à ceux de la natation sportive régie par la FINA. En effet, nous sommes passés d'une 


\section{eJRIEPS 26 avril 2012}

distance de $50 \mathrm{~m}$ à celles de $100 \mathrm{~m}, 400 \mathrm{~m}, 1500 \mathrm{~m}$, en nage libre et à celles de $100 \mathrm{~m}$ et de $200 \mathrm{~m}$ en brasse, en dos crawlé, en papillon ainsi que le $200 \mathrm{~m} 4$ nages. Sans parler de l'augmentation des exigences chronométriques qui, au niveau du CAPEPS (1970-1985), étaient équivalentes à l'époque pour obtenir une note de 20/20 pour les jeunes gens à un niveau international - 100m NL 50"6, 100m brasse 1'04"1 - et national pour les jeunes filles - 100m NL 59"3, 100m brasse 1'15"1. Ce constat témoigne de la volonté de sportiviser davantage les épreuves pratiques de natation sur la base des éléments culturels propres à la natation sportive extrascolaire d'obédience olympique. Notons que les exigences chronométriques pour les élèvesmaîtres, puis les élèves-professeurs-adjoints d'EPS, ont correspondu (1973-1985) à un niveau de pratique en natation nettement plus faible que celui défini pour les élèves-professeurs dans toutes les spécialités et distances nagées. C'est à compter de 1990 que les épreuves de natation du CAPEPS ont été réduites à un 100m de spécialité ou un 200m 4 nages et que les exigences chronométriques ont été abaissées avec en toile de fond la suppression des APS dites de polyvalence et d'option. Cette tendance est à mettre en perspective avec le rattachement de l'EPS à l'Éducation nationale en 1981 et, de manière consubstantielle, à la volonté de vouloir afficher une identité et des attributs plus scolaires que performatifs pour des raisons stratégiques de pérennisation d'une EP scolaire dans et à l'école.

Deuxièmement, en ce qui concerne l'évolution de la nature des savoirs théoriques sur la natation, on peut affirmer qu'il y a eu tant chez les élèves-professeurs que chez les élèves-maîtres et élèves-professeurs-adjoints d'EPS une permanence d'exigences curriculaires autour des aspects historiques, réglementaires et technologiques en liaison avec les nages utilitaires entre 1945 et 1960, et avec les quatre nages sportives durant toute la seconde moitié du XXe siècle. Enfin, s'agissant des savoirs d'action, il apparaît clairement que le législateur, en cohérence avec les exigences fixées par les Instructions officielles de l'EPS (1945, 1959, 1962, 1967, 1985-1986), a continuellement cherché à recruter des profils de professionnalité d'enseignant-e d'EPS en capacité de conduire des leçons de natation élémentaire, axée principalement sur l'apprentissage d'un savoir nager minimal et sécuritaire, voire sportive en fonction de l'âge des élèves, de leur niveau de pratique et de leurs difficultés dans cette activité sans distinction de sexe. On peut préciser qu'à partir du début des années 70, la parole d'autorité a demandé à ce que tous les futurs enseignant-e-s d'EPS (certifié-es, maîtres-ses, PA d'EPS) soient 
eJRIEPS 26 avril 2012

aptes à établir à l'endroit de l'enseignement des APS, une programmation, un programme et une progression de séances en liaison avec la Programmation des APS dans les établissements scolaires du second degré du 3 mai 1967 et la circulaire du 19 octobre 1967 relatives aux Instructions officielles en EPS.

Si l'identification de l'ensemble des savoirs natatoires exigés par la parole d'autorité est relativement aisée à mettre en lumière à partir de l'exégèse des textes officiels qui ont encadré les concours de recrutement d'enseignant-e-s d'EPS, en revanche, que savons-nous de la nature exacte des savoirs natatoires qui furent concrètement dispensés, entre 1945 et 1995, à des étudiants en EPS dans différents centres de formation (CREPS, IREPS, ENSEP, UEREPS, UFRSTAPS), en fonction de leurs ressources humaines et matérielles (piscines) locales. 
eJRIEPS 26 avril 2012

Tableau VI. Ensemble des savoirs natatoires dispensés en formation initiale (CAPEPS, maîtrise d'EPS et PA EPS) 1945-1995.

\begin{tabular}{|c|c|c|c|c|c|}
\hline $\begin{array}{c}\text { Savoirs } \\
\text { aquatiques }\end{array}$ & $\begin{array}{c}\text { ENSEPS } \\
1945-1975\end{array}$ & $\begin{array}{c}\text { CREPS } \\
1945-1984\end{array}$ & $\begin{array}{c}\text { IREPS } \\
1945-1969\end{array}$ & $\begin{array}{c}\text { UEREPS } \\
1969-1984\end{array}$ & $\begin{array}{c}\text { UFRSTAPS } \\
1984-1995\end{array}$ \\
\hline $\begin{array}{l}\text { Savoirs } \\
\text { pratiques }\end{array}$ & $\begin{array}{c}\text { Les } 3 \text { ou } 4 \text { nages } \\
\text { sportives, les départs, } \\
\text { les virages } \\
\text { plongeons sportifs, } \\
\text { plongeon des } 3 \mathrm{~m}, \\
\text { sauvetage aquatique. }\end{array}$ & $\begin{array}{c}\text { Les } 4 \text { nages } \\
\text { sportives les } \\
\text { départs, les virages } \\
\text { plongeon des } 3 \mathrm{~m} \text {, } \\
\text { sauvetage aquatique. }\end{array}$ & $\begin{array}{c}\text { Les } 3 \text { ou } 4 \text { nages } \\
\text { sportives les } \\
\text { départs, les virages, } \\
\text { plongeon des } 3 \mathrm{~m} \text {, } \\
\text { sauvetage } \\
\text { aquatique. }\end{array}$ & \begin{tabular}{|} 
Les 4 nages \\
sportives les départs, \\
les virages plongeon \\
des $3 \mathrm{~m}$, sauvetage \\
aquatique. Water-polo, \\
natation synchronisée.
\end{tabular} & $\begin{array}{c}\text { Les } 4 \text { nages } \\
\text { sportives } \\
\text { les départs, les } \\
\text { virages } \\
\text { sauvetage aquatique, } \\
\text { water-polo, natation } \\
\text { synchronisée. }\end{array}$ \\
\hline $\begin{array}{c}\text { Savoirs } \\
\text { théoriques }\end{array}$ & $\begin{array}{l}\text { Aspects historiques, } \\
\text { réglementaires, } \\
\text { techniques, } \\
\text { bio-mécaniques } \\
\text { des nages sportives }\end{array}$ & $\begin{array}{l}\text { Aspects historiques, } \\
\text { réglementaires, } \\
\text { techniques, } \\
\text { bio-mécaniques } \\
\text { des nages sportives }\end{array}$ & $\begin{array}{l}\text { Aspects historiques, } \\
\text { réglementaires, } \\
\text { techniques, } \\
\text { bio-mécaniques } \\
\text { des nages sportives }\end{array}$ & $\begin{array}{l}\text { Aspects historiques, } \\
\text { réglementaires, } \\
\text { techniques, } \\
\text { bio-mécaniques } \\
\text { des nages sportives }\end{array}$ & $\begin{array}{l}\text { Aspects historiques, } \\
\text { réglementaires, } \\
\text { techniques, } \\
\text { bio-mécaniques } \\
\text { des nages sportives }\end{array}$ \\
\hline $\begin{array}{l}\text { Savoirs } \\
\text { d'action }\end{array}$ & $\begin{array}{c}\text { Apprendre à enseigner } \\
\text { la natation selon la } \\
\text { méthode de Schœbel } \\
\text { (1945-1966) puis } \\
\text { conception moderne et } \\
\text { synthétique : } \\
\text { familiarisation, } \\
\text { ERP, nages sportives, } \\
\text { les } \\
\text { virages et départs, les } \\
\text { plongeons sportifs } \\
\text { (1967-1975) } \\
\text { Pédagogie appliquée } \\
\text { avec } \\
\text { de vrais élèves } \\
\text { années } 60\end{array}$ & $\begin{array}{c}\text { De rien à quelques } \\
\text { rudiments pédagogiques } \\
\text { donnés en salle de } \\
\text { cours ou sur polycopiés } \\
\text { à de la pédagogie } \\
\text { appliquée avec des } \\
\text { élèves ou des } \\
\text { camarades de promotion } \\
\text { (CREPS Bordeaux) } \\
\text { Conception Catteau: } \\
\text { ERP, nages sportives, } \\
\text { virages, départs... }\end{array}$ & $\begin{array}{c}\text { Quelques rudiments } \\
\text { pédagogiques donnés } \\
\text { en salle de cours ou } \\
\text { sur polycopiés } \\
\text { Conceptions : Schœbel } \\
\text { puis Catteau : ERP, } \\
\text { nages sportives, } \\
\text { virages, départs ... }\end{array}$ & $\begin{array}{c}\text { Formation } \\
\text { pédagogique donnée } \\
\text { en salle de cours } \\
\text { et stage en } \\
\text { établissement scolaire } \\
\text { (4e année) : } \\
\text { Conceptions : Catteau } \\
\text { ERP, nages, virages } \\
\text {; et Vadepied, } \\
\text { Azémar (années } 80 \text { ) } \\
\text { (contre l'invasion } \\
\text { technicienne) }\end{array}$ & $\begin{array}{c}\text { Formation } \\
\text { pédagogique donnée } \\
\text { en salle de cours et } \\
\text { stage en } \\
\text { établissement } \\
\text { scolaire (4e année) : } \\
\text { conception Catteau : } \\
\text { ERP, nages, } \\
\text { virages, et Vadepied, } \\
\text { Azémar (années } 80 \text { ) } \\
\text { contre l'invasion } \\
\text { technicienne }\end{array}$ \\
\hline
\end{tabular}

D'après le tableau $\mathrm{VI}$, on peut écrire que les savoirs pratiques transmis à l'ensemble des enseignant-e-s d'EPS de la population étudiée ont pour l'essentiel gravité (19451995) autour de l'acquisition, voire du perfectionnement pour les optionnaires natation, des quatre nages sportives et de leurs virages et départs respectifs dans l'optique de préparer au mieux l'épreuve de parcours chronométré en natation et des démonstrations de techniques de nage (1945-1981). Ce qui semble avoir varié d'un centre à un autre, entre 1945 et la fin des années 70, c'est surtout les conditions d'apprentissage et de perfectionnement en natation. En l'espèce, on note des différences, entre ceux qui avaient, à l'ENSEP, une piscine à disposition à l'année pour s'entraîner régulièrement et ceux qui devaient se déplacer, comme au CREPS de Strasbourg, pour suivre un ou deux stages de natation par an ou rejoindre, au CREPS de Houlgate, toutes les semaines en autocar une piscine à une quarantaine de kilomètres. Mais, on remarque qu'au cours des années 80 et 90 les étudiant-e-s 
eJRIEPS 26 avril 2012

en EPS ont généralement eu de meilleures conditions en pratique natation du fait de l'augmentation de la construction de piscines chauffées à l'année telles que des piscines universitaires, des piscines municipales ou des piscines de type Caneton, Iris ou Tournesol issues de l'opération nommée les 1000 piscines au début des années 70 (Jean-Baptiste Grosborne). S'agissant de la nature des savoirs théoriques, nous constatons qu'ils ont continuellement (1945-1995) reçu dans tous les centres de formation des cours sur les aspects historiques, règlementaires et technologiques à propos des nages sportives et de leurs départs et virages respectifs. Pour ce qui relève des savoirs d'action nous observons d'importantes disparités. Effectivement, si certains d'entre eux ont pu bénéficier en fonction des ressources propres à chaque centre de formation d'une pédagogie appliquée en natation, comme à l'ENSEP avec de vrais élèves et/ou des camarades de promotion au CREPS de Bordeaux, d'autres n'ont rien reçu ou quelques rudiments pédagogiques et/ou didactiques alors donnés en salle de cours ou sous la forme de polycopiés pour apprendre à enseigner la natation. Or, les concours de recrutement d'enseignant-e-s d'EPS ont parfois comporté des épreuves de pédagogie pratique à l'endroit de l'enseignement d'APS comme la natation durant les années 50 et 60 . Ajoutons que d'une manière générale, si les conditions d'apprentissage des savoirs en natation ont pu largement varier d'un centre à un autre, entre 1945 et le milieu des années 80 , et bien, à compter de la fin des années 80 , on a pu observer, à l'échelon national, une homogénéisation positive des conditions matérielles et humaines de formation en natation en raison notamment de la construction de piscines rendues disponibles à proximité des centres de formation.

\section{2. Les formations natatoires dans le champ sportif}

Si les enseignant-e-s d'EPS de la population étudiée ont généralement pu acquérir au cours de leurs études en EPS des savoirs sur la natation et son enseignement, par ailleurs, certains d'entre eux (tableau III) ont pu également s'approprier des savoirs natatoires au contact de formations tels que le diplôme de maître nageur sauveteur (MNS) datant de 1951, le diplôme d'entraîneur de natation (1954), le Brevet d'État d'entraîneur de natation sportive (1966), le Brevet d'État option natation à trois degrés (1966-1983) et enfin du BEESAN (1984-1995). Que dire alors globalement de la nature des savoirs pratiques, théoriques, d'action et d'expérience acquis sur la natation par le bais de ces formations diplômantes ? 


\section{eJRIEPS 26 avril 2012}

5. 2. 1. Du diplôme de MNS au BEESAN 1951-1995

En 1951 l'État français décida de mettre en place le diplôme de MNS (Arrêté du 31 juillet 1951) eu égard à l'évolution des pratiques physiques sociales en matière de natation. II s'agissait alors d'encadrer les pratiques corporelles aquatiques pour des raisons qui tenaient à la sécurité des baigneurs et des nageurs et de contrôler l'enseignement, contre rémunération, de la natation dans des établissements publics à entrée payante. En 1985, ce diplôme devint le BEESAN (option natation) qui donnait alors à ces titulaires les prérogatives pour la surveillance de la baignade, l'enseignement de la natation et l'entraînement des nageurs à la compétition. Par rapport au CAPEPS et à la maîtrise d'EPS, il nous faut indiquer qu'entre 1945 et 1964 les candidat-e-s à ces deux concours ont pu obtenir une équivalence au diplôme de MNS en se présentant, au moment des examens pratiques, à une épreuve d'apnée composée de trois séries de 15 secondes alors rendue facultative, sauf entre 1954 et 1955, en plus des épreuves de sauvetage aquatique, de réanimation et de soins aux noyés. Ainsi, entre 1951 et 1964, la quasi-totalité des enseignant-e-s d'EPS de la population étudiée obtinrent le titre de MNS sans avoir eu besoin de se former en dehors de leurs études en EPS. C'est pourquoi, ils n'ont généralement pas acquis d'autres ou de nouveaux savoirs natatoires que ceux donnés en formation initiale pour passer le MNS. Mais, avec l'arrêté du 14 décembre 1964, cette épreuve d'apnée fut supprimée ce qui obligea alors les candidat-e-s au CAPEPS et la maîtrise d'EPS de se présenter aux sessions normales de l'examen de MNS en l'absence dorénavant d'équivalence possible. Quels sont alors les savoirs natatoires qui furent concrètement acquis par ceux et celles qui suivirent une préparation au diplôme de MNS puis au BEESAN entre 1965 et 1995 ?

Sans trop vouloir détailler, nous pouvons écrire qu'entre 1965 et 1995 les enseignant-e-s d'EPS qui ont suivi sous des formes variées telles que des stages bloqués et des formations modulaires annualisées, une préparation au diplôme de MNS, puis au BEESAN sous l'égide du Ministère de la Jeunesse et des Sports avec des Conseillers Techniques Régionaux en natation, ont généralement pu enrichir leurs savoirs sur l'activité natation au travers de ses aspects historiques, réglementaires, technologiques, bio-mécaniques, didactiques et pédagogiques. Parfois, on leur a même donné en charge des groupes de nageurs de compétition afin de vérifier leurs savoirs et leurs compétences à conduire une ou des séances 


\section{eJRIEPS 26 avril 2012}

d'entraînement intégrées dans une planification annuelle. En outre, ces diverses formations leur ont permis d'acquérir de nouveaux savoirs sur l'hygiène des piscines, la réglementation des baignades, la réanimation.

5. 2. 2. Du diplôme d'entraîneur de natation sportive au Brevet d'État 1954-1995

À côté du diplôme de MNS, certains témoins de la population étudiée ont, entre 1954 et 1984, suivi durant leur socialisation secondaire une formation pour obtenir le diplôme d'entraîneur de natation. Indiquons que le premier Brevet d'État (BE) de natation sportive avait vu le jour en 1954 (arrêté du 19 janvier 1954). En 1956, il devient le Brevet d'État d'entraîneur de natation sportive (loi du 6 août 1963, arrêté du 6 mai 1966) jusqu'à la mise en place progressive du Brevet d'État à trois degrés option natation (1974-1979). D’une manière générale, entre 1954 et 1985, les contenus pratiques exigés ont porté sur des épreuves chronométrées au choix parmi les quatre nages sportives, des démonstrations de techniques de nage sportive, de virage et de départ. Au niveau des épreuves écrites et orales, elles devaient permettre d'apprécier d'une part, les connaissances des candidat-e-s sur les techniques de nage (virage, départ) au travers de leurs aspects historiques, réglementaires, technologiques, ainsi que sur l'organisation du sport en France, sur la gestion d'une compétition et les fonctions inhérentes au rôle d'officiel en tant que juge ; et, d'autre part, sur des savoirs d'action relatifs à l'enseignement et à l'entraînement en natation à l'endroit de la physiologie de l'effort et de la préparation physique. Pour ceux qui avaient entrepris de passer le BE natation $2^{e}$ degré, puis éventuellement le $3^{e}$ degré (Arrêté du 8 mai 1974), ils ont dû se former pour officier lors de compétitions européennes ou internationales de natation et piloter des actions de formation pour les cadres de la Fédération Française de Natation (FFN). Enfin, au niveau du $\mathrm{BE} 3^{\mathrm{e}}$ degré, ils devaient monter leurs capacités à conduire des stages nationaux d'entraînement pour des nageurs et nageuses sélectionnés en équipe nationale à partir de séjours de formation à l'étranger comme aux USA par exemple.

Les témoins de la population étudiée titulaires d'un BE natation $\left(1^{\mathrm{er}}, 2^{\mathrm{e}}\right.$ ou $3^{\mathrm{e}}$ degré $)$ ont souvent suivi des cours sous la forme de stages à l'Institut National du Sport entre 1946 et 1974, puis à l'Institut National du Sport et de l'Education Physique au cours de la période de 1975 à 1995, au contact d'entraîneurs nationaux comme Georges Garret ou Lucien Zins et d'enseignants d'EPS spécialistes de natation à 


\section{eJRIEPS 26 avril 2012}

l'instar de Marc Menaud. Ces temps de formation leur ont permis de compléter leurs savoirs pratiques et théoriques natatoires acquis durant leurs études en EPS et d'enrichir, selon eux, considérablement leurs savoirs d'action à l'endroit de l'entraînement en natation et de la formation des cadres de la FFN. Au final, il est évident que les formations natatoires diplômantes développées au sein du champ sportif ont donné l'occasion à leurs impétrants d'enrichir tant et plus leur culture sur la natation et en particulier leurs savoirs sur l'entraînement et la préparation physique des nageurs de compétition. Bien souvent, ceux qui ont obtenu le BE natation $2^{\mathrm{e}}$ ou $3^{e}$ degré ont conduit ensuite, durant leur carrière, des actions de formation en direction des personnels tels que les instituteurs, les enseignant-e-s d'EPS et les cadres de la FFN.

5. 3. L'univers professionnel en EPS et l'hégémonie de la conception de Raymond Catteau

$\mathrm{Si}$, comme nous venons de le voir, certains enseignant-e-s d'EPS de la population étudiée ont pu étoffer, par rapport à leur formation initiale, leur niveau d'expertise et d'expérience en natation par le truchement de formations sportives fédérales, en revanche, tous ont pu compter sur leur univers professionnel pour y trouver un ensemble de savoirs natatoires afin, selon eux de les aider à concevoir et à conduire leur enseignement de la natation. Quels sont alors les principaux savoirs en natation qui ont été diffusés dans leur univers professionnel et appris par le bais de la formation continue et l'auto-formation?

\section{3. 1. La Revue EPS}

Selon leurs déclarations, la Revue EP.S est celle qui a été manifestement la plus citée ( $\mathrm{n}=93)$, à côté de la Revue Hyper-EPS ( $\mathrm{n}=11)$ ou encore la Revue Dire en APS $(n=9)$, comme étant une source d'informations sur la natation et son enseignement. 
eJRIEPS 26 avril 2012

Tableau VII. Liste des revues professionnelles les plus citées par la population étudiée $(n=143)$.

\begin{tabular}{|l|c|}
\hline \multicolumn{1}{|c|}{ Liste des revues professionnelles citées } & Quantité de fois citées \\
\hline Revue EPS (1950) & 93 \\
\hline Hyper EPS, Bulletin de l'Amicale ENSEP (1936) & 9 \\
\hline 1.1.1.1.1.1.1.1 Revue Dire en APS CPS-FSGT (1981) & 5 \\
\hline Spirales (1983) & 4 \\
\hline 1.1.1.1.1.1.1.2 Sport et Vie (1990) & 3 \\
\hline Cahiers EPS académique de Nantes (1989) & 2 \\
\hline 1.1.1.1.1.1.1.3 Cahiers pédagogiques (1945) & 127 \\
\hline Total & \\
\hline
\end{tabular}

Notons que parmi toutes les revues ici citées, seule la Revue EPS a été constamment publiée entre 1950 et 1995. Concernant donc l'activité natation, voyons à partir d'une analyse de contenu, les savoirs qui ont été diffusés dans les colonnes de cette revue professionnelle (1950-1995). Pour ce qui est des savoirs d'action, on peut écrire que c'est incontestablement la conception dite moderne et synthétique de l'enseignement de la natation de Raymond Catteau et de ses consorts tels que M. Menaud, Paul-Raymond Guilbert, Jean Vivensang, qui a dominé cet espace de diffusion de savoirs didactiques et pédagogiques sur les APSA. Rappelons que la conception «catteaussienne » s'est inscrite dans le prolongement de l'approche naturelle développée dès l'entre-deux-guerres par Gilles de Villepion, Raymond Siener et Émile Schœbel. Ces deux conceptions, naturelle et synthétique, étaient principalement axées sur l'acquisition de la flottaison naturelle du corps humain sans aide matérielle, sur l'apprentissage de la respiration aquatique et de la glisse par la posture aquatique dite fusiforme (Siener, 1948), autrement dit sur le triptyque équilibre-respiration-propulsion (ERP). Cette domination de la conception évolutive de R. Catteau (Érard \& Catteau, 2008) ne saurait toutefois faire oublier qu'il a existé d'autres manières de penser et d'organiser l'enseignement de la natation en EPS durant la seconde moitié du XXe siècle. En effet, à côté de l'hégémonique conception «catteaussienne » finalisée par l'apprentissage des quatre nages 
eJRIEPS 26 avril 2012

sportives et de l'entraînement en natation, les enseignant-e-s d'EPS ont pu toutefois trouver dans la revue EP.S d'un côté, des conceptions béhavioristes pour l'enseignement de la brasse utilitaire (Ernest Trotzier) et des nages sportives (Claude Dubois, Jean-Pierre Robin, Étienne Bordat) et, de l'autre côté, des conceptions constructivistes portées par Alain Vadepied, Jacques Vallet, Guy Azémar, Pierre Arnaud dans lesquelles l'aspect culturel des apprentissages natatoires fut relayé à un second plan craignant à l'époque (années 70-80) l'invasion de la technique en EPS et la réification du corps des élèves en les assujettissant aux seules techniques de nage sportive. Effectivement si, au début des années 50 , on a continué à vanter l'efficacité dans la Revue EP.S (1951) des méthodes d'enseignement par suspension d'élèves - la potence (E. Trotzier) - pour apprendre de manière mécanique la brasse, en revanche, au début des années 70 , les conceptions centrées sur l'activité adaptative du sujet confronté aux contraintes et aux ressources de l'eau ont émergé en reléguant à un second plan l'apprentissage des techniques de nage sportive. Ces diverses conceptions de l'enseignement de la natation illustrent plus globalement les rapports parfois conflictuels entre les tenants d'EPS axée principalement sur des apprentissages fondés sur des gestes socialement et culturellement fondés comme les nages sportives (R. Catteau); et les laudateurs d'une natation scolaire ancrée essentiellement sur la capacité des élèves à adapter leur motricité de terrien dans des situations aquatiques d'auto-apprentissage assez éloignées des modèles techniques issus de la natation sportive (Vallet, 1969, 1974; Azémar, 1974 ; Vadepied, 1976, 1978) pourtant programmés dans les textes officiels de l'EPS (IO 1967, IO 1985-1986). Enfin, précisons que cette revue professionnelle leur a régulièrement communiqué d'une part, des savoirs sur les résultats sportifs en natation en liaison avec les Jeux Olympiques et les Championnats de Monde et, d'autre part, sur l'évolution des techniques de nage sportive sous la forme d'analyses descriptives, adossées à des kinogrammes, de la gestualité des nageurs experts comme étant la référence à enseigner aux élèves en adéquation avec le curriculum prescrit par les textes officiels en EPS entre 1967 et 1995.

5. 3. 2. Les ouvrages, les vidéos natatoires et la formation professionnelle continue

À côté de le Revue EP.S, les témoins nous indiquent qu'ils ont pu également compter sur des ouvrages et des vidéos de natation étudiés durant leur socialisation 


\section{eJRIEPS 26 avril 2012}

secondaire, c'est-à-dire soit en formation initiale et/ou en FPC ou par auto-formation. Ils nous indiquent que cet ensemble de publications a constitué en quelque sorte un réservoir de réponses pratiques possibles pour les aider dans la conduite de leur enseignement de la natation en fonction de l'évolution du curriculum formel, de leurs propres savoirs expérientiels et des conditions locales liées aux publics cibles, aux choix didactiques d'équipe EPS et aux conditions matérielles.

Tableau VIII. Liste des principaux ouvrages de natation étudiés par la population étudiée $(n=143)$.

\begin{tabular}{|c|c|}
\hline Ouvrages & $\begin{array}{l}\text { Nombre de } \\
\text { fois cités }\end{array}$ \\
\hline $\begin{array}{l}\text { R. Catteau, \& G. Garoff, (1968). L'enseignement de la natation. Paris : Éditions } \\
\text { Vigot. }\end{array}$ & 85 \\
\hline $\begin{array}{l}\text { E. Schœbel, (1947). Précis de natation scolaire. Carnets de l'éducation physique } \\
\text { et des sports. Paris : Bourrelier. }\end{array}$ & 18 \\
\hline $\begin{array}{l}\text { J. Vivensang, (1977). La Pédagogie moderne de la natation. } 3^{\mathrm{e}} \text { Édition, Paris: } \\
\text { Éditions Chiron Sports. }\end{array}$ & 17 \\
\hline M. Menaud, \& L. Zins, (1965). La natation. Paris : Éditions Amphora. & 15 \\
\hline $\begin{array}{l}\text { C. Dubois, \& J.-P. Robin, (1985). Natation. Collection « De l'école aux } \\
\text { associations ». Paris : Éditions EP.S. }\end{array}$ & 13 \\
\hline $\begin{array}{l}\text { J.-E. Counsilman, (1975). La natation. Tome premier Les Techniques. Tom } \\
\text { second, L'entraînement. Traduction de The science of swimming. Paris : Éditions } \\
\text { Chiron. }\end{array}$ & 13 \\
\hline $\begin{array}{l}\text { A. Vadepied, (1976). Laissez l'eau faire. Paris, Éds Scarabée ; (1978). Les eaux } \\
\text { troublées. Paris : Éditions Scarabée. }\end{array}$ & 11 \\
\hline P.-R., Guilbert (1968). La natation d'aujourd'hui. Paris : Éd. Borneman. & 9 \\
\hline
\end{tabular}


eJRIEPS 26 avril 2012

Tableau IX. Liste des vidéos natatoires les plus citées par la population étudiée $(n=143)$.

\begin{tabular}{|l|c|}
\hline \multicolumn{1}{|c|}{ Liste des vidéos les plus citées } & $\begin{array}{c}\text { Quantité de } \\
\text { fois citées }\end{array}$ \\
\hline Digne, Dingue, D'eau, de Raymond Catteau, 1979, INSEP. & 19 \\
\hline $\begin{array}{l}\text { Natation. Les nages simultanées. Les nages alternées. de J.-P. Robin \& C. } \\
\text { Dubois, INSEP, 1986. }\end{array}$ & 5 \\
\hline Nouveau départ, J. Ribaud, INSEP, 1983. & 5 \\
\hline Films australiens, analyses des techniques de nage sportive, années 60 & 3 \\
\hline Les Eaux Claires, 1947. & 40 \\
\hline Total & \\
\hline
\end{tabular}

À la lecture des tableaux VIII et IX, il apparaît clairement que dans la continuité de la conception dite naturelle de l'enseignement de la natation développée par É. Schœbel (1942), celle de R. Catteau semble avoir été celle qui fut la plus massivement travaillée durant leurs études en EPS et diffusée dans leur univers professionnel de la fin des années 60 jusqu'au début des années 90 . Notons que les pratiques pédagogiques déclarées et rapportées à propos de l'enseignement de la natation en EPS (1945-1995) portent l'empreinte de ces deux conceptions dominantes à côté des conceptions béhavioristes (Auvray, 2011).

Enfin, les données que nous avons pu recueillir concernant la FPC montrent que dans la majorité des cas les savoirs natatoires qui ont été transmis, dès la fin des années 60, ont largement gravité autour de la conception et des propositions pratiques de R. Catteau et de ses consorts, tous enseignants d'EPS et formateurs en natation, à savoir M. Menaud à I'INS, P.-R. Guilbert à l'ENSEPS, Jean Vivensang au CREPS de Bordeaux, Patrick Schmitt chez les instituteurs. Les éléments récurrents donnés généralement en FPC ont porté sur la familiarisation aquatique sans aide matérielle, sur l'acquisition du triptyque équilibre-respiration-propulsion et l'affinement des prises d'informations ainsi que sur l'apprentissage des nages sportives, des virages et des départs de spécialité, et enfin sur l'initiation à l'entraînement en natation. Or, à l'échelon individuel et de manière diachronique (1945-1995) et synchronique, quels que soient leurs sensibilités, leurs conceptions de l'EPS défendues (Lorca, 2002), leurs profils d'expertise et d'expérience en natation et leurs 
eJRIEPS 26 avril 2012

rapports aux savoirs à enseigner entre l'appétence et le déplaisir, les enseignant-e-s d'EPS de la population étudiée déclarent qu'ils ont tous généralement fait évoluer leur enseignement de la natation d'une logique d'apprentissage qualifiée, par euxmêmes, de techniciste vers une logique plus axée sur l'adaptabilité et l'affectivité des élèves en situation potentiellement anxiogène sans toutefois perdre de vue l'acquisition de modes de déplacements ancrés sur les nages sportives.

\section{Conclusion}

Le chemin qui mène à la formalisation historique (1945-1995) des savoirs acquis sur la natation chez une population hétérogène d'enseignant-e-s d'EPS ayant vécu différentes trajectoires biographiques est long et suppose d'avoir eu recours à des astuces pour trouver et mobiliser auprès de ces acteurs-témoins des archives privées et semi-officielles. De l'ensemble de notre corpus, il en ressort donc une histoire (1945-1995) des savoirs qui ont été transmis sur la natation activité cardinale en EPS. En l'espèce, l'investigation des savoirs natatoires qui furent exigés par l'État recruteur et réellement diffusés à des étudiant-e-s en EPS scolarisé-e-s dans diverses structures de formation (CREPS, IREPS, ENSEPS, UEREPS, UFRSTAPS) pour y préparer soit le CAPEPS, la maitrise ou le PA d'EPS, montre qu'il y a existé à la fois des permanences et des fragmentations tant au niveau de la nature des contenus dispensés que des moyens pédagogiques employés localement pour les transmettre. Pour autant, les savoirs natatoires concrètement donnés en formation initiale n'ont jamais été (1945-1995) en totale dissonance avec les exigences fixées par l'État recruteur. Car, il est évident que pour l'ensemble des centres de formation ici analysés, l'enjeu résidait aussi dans la nécessité de préparer au mieux leurs étudiant-e-s aux concours de recrutement d'enseignant-e-s pour afficher leur efficacité et leur bonne santé en matière de formation. Toutefois, entre 1945 et 1985 , on constate d'importantes variations au niveau des conditions de la pratique natation et du sauvetage aquatique en fonction des ressources propres à chaque structure de formation qui semblent s'être toutefois atténuées au fil du temps (1985-1995) du fait de la construction de piscines universitaires ou municipales disponibles à proximité de ces instituts de formation. On note que dans certains centres, comme au CREPS de Bordeaux, la formation à la pédagogie de la natation y fut plus poussée que dans d'autres structures, en raison manifestement de la présence, durant les années 7080 , de formateurs enseignants d'EPS comme J. Vivensang et Paul Guerpillon très 


\section{eJRIEPS 26 avril 2012}

attachés à cette partie des études. Par ailleurs, si l'univers professionnel de l'EPS constitue un réservoir de savoirs disponibles pour compléter et enrichir la formation initiale, dans le même temps, il contribue à façonner et à homogénéiser la rationalité pratique des acteurs «ordinaires » de l'EPS autour de conceptions pédagogiques en natation rendues manifestement dominantes (R. Catteau) au moment où, au tournant de 1967, la paorle d'autorité avait rejoint la parole sacrée, c'est-à-dire celle des conceptions. En atteste le contenu de la Programmation des APS du 3 mai 1967 qui, pour la partie natation, correspondait à la conception moderne et synthétique de $\mathrm{R}$. Catteau bien que ce dernier n'ait pas participé aux commissions qui eurent en charge à l'époque la rédaction de cette Programmation souhaitée et pilotée par l'Inspection générale de l'EPS. Enfin, la relative disparité des savoirs transmis sur l'activité natation à des étudiant-e-s en EPS se retrouve également au niveau de l'enseignement réel de la natation en EPS (Auvray, 2010) pour des raisons essentiellement identiques à savoir localement, les conditions matérielles, les choix pédagogiques d'équipe, l'implantation géographique des établissements scolaires (mer, ville, campagne), les conceptions de l'EPS et de l'enseignement de la natation portées par les enseignant-e-s. Plus généralement, nos résultats nous conduisent à nous questionner sur l'impact de la disparité des conditions locales de formation initiale chez les futurs enseignant-e-s d'EPS d'une part, vis-à-vis de l'égalité des chances de réussite aux concours de recrutement et, d'autre part, sur la nature de leurs pratiques pédagogiques développées au cours de leur carrière. Finalement, ce travail de recherche socio-historique aura permis de mettre en lumière, chez des enseignant-e-s " ordinaires " de l'EPS, une histoire culturelle et une épistémologie de leurs savoirs en natation acquis à l'aune de leur cheminement biographique personnel et professionnel et les ressorts de leur rationalité pratique, axiologique et psychologique à l'endroit de l'enseignement de la natation scolaire.

\section{Sources}

1. Bulletin Officiel de l'Éducation nationale, période 1945-1995, sur les programmes du CAPEPS, de la maîtrise d'EPS et du PA d'EPS.

2. Archives sur des cours théoriques et pratiques en formation initiale

- Au CREPS de Dinard (Ille-et-Vilaine) : Référence G.31.e.21, "Plan de cours sur la technique ", Auteur, J.-M. Castillo, année 1985-1986 ; Référence G.31.g.34, 
eJRIEPS 26 avril 2012

"Natation », Auteurs, Decourt et collectif, PA2 année 1982-1983 ; Référence G.31.e.18, «Dossier Natation», Auteur, Bonnet Ronald, PA2 année 1985 ; Référence G.31.g.30, "Compte rendu pédagogique ", Auteurs, Guisgand et Van. Ighem, PA2 1981.

3. Archives privées ou semi-officielles

- En formation initiale

Notes de cours théoriques et pratiques de Jules Benoît, professeur certifié d'EPS, né en 1939, promotion ENSEP 1958-1961 sur : « Les nages sportives »; " La natation élémentaire-sportive-utilitaire » "Les plongeons sportifs et utilitaires »; " Noyade et sauvetage. " "L'enseignement de la natation élémentaire ». Compte rendu d'un ensemble de séances pratiques avec M. Schœbel, octobre 1959.

Exposé de M. Millet, élève de $2^{\mathrm{e}}$ année ENSEP promotion 1958-1961, sur la “ Natation » et «Les effets physiologiques de la natation ».

Note de cours théoriques et pratiques sur la natation et son enseignement de $\mathrm{M}$. Hervé Gicquel, agrégé d'EPS, CREPS de Bordeaux 1967-1970.

Maquettes des études de l'UEREPS de Caen entre 1985 et 1993 ; et de l'UEREPS de Lille années 80 .

- En formation continue professionnelle

Bilan " Journée d'informations sur l'enseignement de la natation " pour les professeurs d'EPS à Caen en 1963, document de Jules Benoît.

Stage FPC Natation, "Comment observer l'enfant? " et "Perfectionnement des stagiaires en natation, à Caen, octobre 1974, document de Jules Benoît.

"Schéma d'une démarche d'apprentissage en natation. " Juin 1984, document de Michel Vinzant, né en 1943, professeur certifié d'EPS, CREPS de Toulouse promotion 1961-1962, puis IREPS de Lyon 1962-1965.

"Stage de recyclage en natation des enseignants d'EPS du collège de Canet ", septembre 1988, document de Christine Ghezal, née en 1949, certifiée EPS, CREPS de Dijon, promotion 1970-1973.

\section{Articles dans la revue EPS classés par ordre chronologique}

Comité de rédaction. (1951). Comment le problème de la natation scolaire a été résolu à Tours. Revue EP.S, 5, 10-11.

Comité de rédaction, (1951). Quatre années d'expérience au Havre. Revue EP.S, 11, 36-37. 
eJRIEPS 26 avril 2012

Vallet, J. (1969). L'enfant et l'eau. Revue EP.S, 100, 15-19.

Azémar, G. (1974). Aspects fondamentaux du comportement du jeune enfant dans l'eau. Revue EP.S, 129-130, 23-47.

Arnaud, P., \& Broyer, G. (1979). Les conduites aquatiques du débutant. Analyse et interprétation de la régression des conduites aquatiques chez des élèves du premier cycle du second degré. Revues EP.S, 158,49-59, 159, 70-73, 160, 73-75.

Catteau, R. (1980). Illustration d'une pédagogie nouvelle en natation : Digne, dingue, d'eau. Un film de la FFN. Revue EP.S, 165, 43.

Meslier, J. (1981). Approche du 4 nages. Revue EP.S, 169, 28-31.

Dubois, C., \& Robin, J.-P. (1982). Le papillon une belle nage. Revue EP.S, 173, 2831.

Bordat, É. (1985). Apprentissage du crawl. Une progression en 8 exercices. Revue EP.S, 191, 33-37.

Dubois, C., Robin, J.-P., Darquie, C. (1989). Baccalauréat : le 100m 4 nages. Revue EP.S, 215, 56-56.

5. Ouvrages classés par alphabétique

Beulque, P. \& A. Descarpentries, (1922). Méthode de natation. Tourcoing : Imprimeur Georges Frère.

Catteau, R. \& Garoff, G. (1968). L'enseignement de la natation. Paris : Éditions Vigot.

Dubois, C. \& Robin, J.-P. (1985). Natation. Collection « De l'école aux associations ». Paris : Éditions EP.S.

Guilbert, P.-R. (1973). La natation aujourd'hui. Paris : Éditions Bornemann.

Hébert, G. (1959). Ma Leçon type de natation. Paris : Librairie Vuibert, $6^{\mathrm{e}}$ édition.

Maurice-Boyrie, J.-P. (1946). Nages sportives. Clichy : Paul Dupont.

Menaud, M. \& Zins, L. (1965). La natation. Technique \& entraînement. Paris: Éditions Amphora.

Schœbel, É. (1947). Précis de natation scolaire. Carnets de l'éducation physique et des sports. Paris : Éditions Bourrelier.

Siener, R. (1948). L'enseignement naturel de la natation utilitaire. Tome 1, Technique des nages, Initiation sportive. Paris : Éditions Étienne Chiron.

Schmitt, P. (1989). Nager : de la découverte à la performance. Paris : Éditions Vigot. 
eJRIEPS 26 avril 2012

Vadepied, A. (1976). Laissez l'eau faire. Paris : Éditions Scarabée.

Vadepied, A. (1978). Les eaux troublées. Paris : Éditions Scarabée.

Vallet, J. (1974). Les bébés nageurs. La familiarisation à l'eau source d'éveil $d d u$ jeune enfant. Paris : Éditions Marabout.

Vivensang, J. (1977). La Pédagogie moderne de la natation. 3e Éd., Paris : Éditions Chiron Sports.

\section{Bibliographie}

Arnaud P. (1983). Les savoirs du corps, Éducation physique et éducation intellectuelle dans le système scolaire français. Lyon : Éditions PUL.

Arnaud, P. (1988). Métier : prof de gym. Enfance et jeunesse d'une profession au travers d'un récit autobiographique (1906-1958). Entretiens avec Jean-Louis Charrière. Le Binet Simon, 614, 3-31.

Attali M, \& Saint-Martin, J. (2004). L'EP de 1945 à nos jours. Les étapes d'une démocratisation. Paris : Armand-Colin.

Attali, M. (2006). L'univers professionnel des enseignants d'éducation physique de 1940 à nos jours. Paris : Vuibert.

Auvray, E. (2010). Une histoire des pratiques pédagogiques liées à l'enseignement de la natation scolaire entre 1945-1995 ( $2^{\text {nd }}$ degré). Revue Carrefours de l'éducation, 30, 149-167.

Auvray, E. (2011). Qu'ont-ils appris et enseigné ? L'évolution des curricula formels et réels liés à l'enseignement de la natation scolaire $\left(2^{\text {nd }}\right.$ degré $)$ et les identités professionnelles chez des enseignants d'EPS (1945-1995). Thèse STAPS, Université de Lyon1.

Bertaux, D. (1997). Les récits de vie. Perspective ethnosociologique. Paris : Nathan.

Caritey, B. (2008). Parcours de vie des enseignants d'EPS et diversité des pratiques professionnelles. Dossier EPS, 77. Paris, Revue EPS, 47-58. 
eJRIEPS 26 avril 2012

Chevrel, A. (1988). L'histoire des disciplines scolaires. Réflexions sur un domaine de recherche. Histoire de l'éducation, 38, 9-119.

Clermont Gauthier, Ph.-D. \& al. (1997). Pour une théorie de la pédagogie. Recherches contemporaines sur le savoir des enseignants. Ste Foy: Les presses de l'université de Laval.

Collinet, C. \& Taled, A. (2003). Histoire et historiens en STAPS de 1975 à nos jours, in C. Collinet, (dir.), La recherche en STAPS. Paris : PUF, 131-152.

Defrance, J. (1978). La fortification des corps. Thèse de doctorat en 3e cycle de sociologie, Paris EHESS.

Dourdin, J. (1942). Etude de l'état d'esprit de la population parisienne à l'égard de l'éducation physique et des sports, Paris.

Dubar, C. (2000). La socialisation. Construction des identités sociales et professionnelles. Paris : Armand Colin.

During, B. (1981). La crise des pédagogies corporelles. Paris : Éditions ScarabéeCEMEA.

Érard C. \& Catteau A. (2008). Les trois modèles d'analyse de l'enseignement de la natation de Raymond Catteau (1950-2006) : influences pédagogiques et scientifiques. In, MUNOZ L. (dir). Usages corporels et pratiques sportives aquatiques du XVIIle au XXe siècle. Paris : L'Harmattan, Tome II, 101-123.

Ferrarotti, F. (1990). Histoire et histoires de vie. La méthode biographique dans les sciences sociales. Collection sociologies au quotidien. Paris: Éditions Méridiens Klincksieck.

Forquin, J.-C. (1989). École et culture, le point de vue des sociologues britanniques. Bruxelles : De Boeck-Wesmael.

Halbwachs, M. (\{1924\}, 1994). Les cadres sociaux de la mémoire. Paris : Albin Michel.

Héry, É. (2005). Les pratiques pédagogiques, objets d'histoire. Carrefours de l'éducation, 19, 93-105. 
eJRIEPS 26 avril 2012

Hurtebize, C. (1981). La formation des cadres pour le sport et pour l'éducation physique. Étude comparée entre la France et la République Démocratique Allemande. Mémoire pour le Diplôme de I'INSEP.

Irlinger, P., Louveau, C., Métoudi, M. (1987). Les pratiques sportives des français. Usages sportifs du temps libéré. Paris, INSEP.

Lahire, B. (1998). L'homme pluriel. Les ressorts de l'action. Paris, Nathan.

Legras, J.-M. (1999). À quoi servent les enseignants d'EPS ? Dossier EPS, 29. A quoi sert l'EPS ? Paris : Éditions EPS, 259-266.

Lorca, P. (2002). Contribution à l'étude des conditions d'émergence de l'EPS comme discipline scolaire. Aperçus de la noosphère. Thèse de Sciences de l'Education, Université Lyon 2.

Mialaret, G. (1991). Pédagogie Générale. Paris : PUF.

Michon, B. (1982). Enquête nationale sur les origines sociales des étudiants en éducation physique et sportive : Identités et stratégies de gestion des propriétés socio-culturelles. Thèse de 3e cycle en sociologie, Paris VII.

Michon, B. (1983). Éléments pour une histoire sociale des enseignants en EPS. STAPS, 8, 21-22.

Michon, B. \& Caritey, B. (1999). Histoire orale d'une profession : les enseignants d'éducation physique. In, P. Arnaud (dir.), Une Histoire de l'éducation physique, Enseignement primaire et secondaire 1880-2000. Spirales, 13-14, 11-45.

Mierzejewski, S. (2005). Le corps académisé. Sur l'accès aux positions universitaires des premiers enseignants-chercheurs en STAPS issus de l'EPS. Science et motricité, 55, 79-99.

Néaumet, P. (1992). L'éducation physique et ses enseignants au XXe siècle. Paris : Amphora.

Pelayo, P. \& Terret, T. (1994). Savoirs et enjeux relatifs à la natation dans les instructions et programmes officiels (1877-1986). STAPS, 33, 79-88.

Perrenoud, Ph. (1993). Curriculum : le formel, le réel, le caché. In J. Houssaye, (dir.). La pédagogie : une encyclopédie pour aujourd'hui. Paris : ESF, 61-76. 
eJRIEPS 26 avril 2012

Postic, M. (1977). Observation et formation des enseignants. Paris : PUF.

Raynal, F. \& Rieunier, A. (1998). Pédagogie : dictionnaire des concepts clés. Paris : ESF.

Ricœur, P. (2000). La mémoire, l'histoire, l'oubli. Paris : Le Seuil.

Robert, A. \& Bouillaguet, A. (1997). L'analyse de contenu. Paris : PUF.

Roux-Perez, T (2011). Identités(s) professionnelles(s) des enseignants. Les professeurs d'EPS entre appartenance et singularité. Paris : Éditions EPS.

Terret, T. (1998). Anciens et nouveaux objets dans l'histoire de l'éducation physique en France. »In, P. Arnaud (dir.), Une Histoire de l'éducation physique, Enseignement primaire et secondaire 1880-2000. Spirales, 13-14, 367-381. 